\title{
Programa Mais Médicos, uma tentativa de solucionar o problema da distribuição médica no território brasileiro
}

\author{
More Doctors Program, an attempt to solve the problem of medical \\ distribution in the Brazilian territory
}

Leonardo Maso Nassar1, João Luiz Passador', Gerson Alves Pereira Júnior²

DOI: 10.1590/0103-1104202113116

RESUMO O objetivo do estudo foi realizar uma revisão integrativa da literatura utilizando a mnemônica População, Conceito e Contexto do Joanna Briggs Institute para mapear os trabalhos que abordaram a distribuição médica no Brasil, com intuito de analisar criticamente o Programa Mais Médicos (PMM), resultando em 453 trabalhos, com 10 artigos selecionados. Os resultados indicam fatores pecuniários e não pecuniários como motivos para a má distribuição brasileira de médicos. Como solução, foi criado o PMM, que provocou grande expansão do número de cursos de medicina - um aumento estimado de $71 \%$ no número de médicos até 2030. Embora haja dificuldades na capacitação técnica e pedagógica docente e de preceptores para supervisionarem essa quantidade abrupta de graduandos, há uma oportunidade para transformar a má qualidade atual do acesso e da assistência prestada à população no Sistema Único de Saúde (SUS). Por outro lado, a falta de um monitoramento contínuo da avaliação da qualidade dos formandos e a falta de investimentos na infraestrutura e na regulação da assistência, bem como da melhor distribuição e qualificação dos programas de residência médica, poderão tornar essa situação, aparentemente vantajosa, uma grande catástrofe (pessoal e profissional), com um mercado de trabalho que pode ter uma enorme oferta de mão de obra pouco qualificada.

PALAVRAS-CHAVE Distribuição de médicos. Consórcios de saúde. Médicos.

1 Universidade de São Paulo (USP), Faculdade de Economia, Administração e Contabilidade de Ribeirão Preto (FEA-RP) - Ribeirão Preto (SP), Brasil. leo.mnassar@gmail.com

2 Universidade de São Paulo (USP), Faculdade de Odontologia de Bauru (FOB) - Bauru (SP), Brasil.

\begin{abstract}
The objective of the study was to carry out an integrative literature review using the mnemonic Population, Concept and Context of the Joanna Briggs Institute, to map the works that addressed the medical distribution in Brazil, in order to critically analyze the More Doctors Program (PMM), resulting in 453 papers with 10 selected articles. The results indicate pecuniary and non-pecuniary factors as reasons for the poor distribution of Brazilian doctors. As a solution, the PMM was created, which caused a great expansion in the number of medical courses, an estimated increase of 71\% in the number of doctors until 2030. Although with the difficulties of technical and pedagogical training of teachers and preceptors to supervise this amount abruptness of undergraduate students, there is an opportunity to transform the current poor quality of access and assistance provided to the population in the Unified Health System (SUS). However, the lack of continuous monitoring of the trainees' quality assessment, lack of investments in infrastructure and regulation of assistance, as well as the better distribution and qualification of medical residency programs, could make this situation apparently advantageous in a major catastrophe (personal and professional) with a labor market that may have a huge supply of low-skilled labor.
\end{abstract}

KEYWORDS Medical distribution. Health Consortia. Doctors. 


\section{Introdução}

A distribuição equitativa de médicos é um problema social e político que afeta quase todos os países atualmente'. Apesar de um número ideal de mé dicos por habitante nunca ter sido estipulado pela Organização Mundial da Saúde (OMS), estima-se que a proporção ideal estaria em uma razão de 2 a 2,5 médicos por 1.000 habitantes $^{2}$. No Brasil, apesar do acesso universal à saúde estar garantido pela Constituição de 1988 e da razão de médicos para cada 1.000 habitantes estar em 2,18, o grande território nacional, parcialmente coberto por florestas densas, é um desafio para a distribuição equitativa de médicos ${ }^{3}$.

Pela análise individual da proporção de médicos em cada Unidade Federativa (UF), é notória a distribuição irregular ${ }^{4}$. Ao longo dos anos, iniciativas como o Programa de Interiorização do Sistema Único de Saúde (Pisus), o Telessaúde, o Projeto Rondon e outras foram realizadas a fim de resolver o problema que ainda persiste, sendo o Programa Mais Médicos (PMM) uma delas5.

Embora várias ações tenham sido desenvolvidas, as instituições de ensino possuem as contribuições mais estudadas pela literatura, com alguns países avançando na interiorização da graduação de medicina com a abertura de cursos em localidades rurais ou remotas ${ }^{6}$. Expandir a oferta de cursos de medicina pelo território nacional é uma prática antiga no Brasil: o Decreto ${ }^{\circ}{ }^{53.642}$, de 28 de Fevereiro de $1964^{7}$, utiliza como justificativa a má distribuição de médicos no território nacional para dobrar a quantidade de vagas nas faculdades de medicina brasileiras e utiliza como exemplo a distribuição no estado do Maranhão, sendo essa a mesma justificativa para expandir a oferta de cursos de medicina presente na Lei $\mathrm{n}^{\circ} 12.871$, de 22 de Outubro de 2013, que institui o $\mathrm{PMM}^{8}$.

É notável que, entre 1964 e 2013, os problemas sejam praticamente os mesmos, e a solução encontrada seja semelhante às práticas antigas que fracassaram. Entretanto, soluções precisam ser encontradas, porque a distribuição equitativa da força de trabalho em saúde, principalmente dos médicos, é fundamental para o País alcançar seus objetivos de desenvolvimento sustentável em saúde e igualdade social ${ }^{9}$. Atualmente, o debate da solução da distribuição passa pela necessidade do aumento do número de médicos no Brasil, envolvendo interesses particulares, ideológicos, partidários ou coorporativos ${ }^{5}$.

Pela análise dos expostos anteriores, é possível perceber a complexidade do problema. Portanto, para investigar com mais profundidade o assunto e analisar PMM com maior embasamento, surge a pergunta norteadora da pesquisa: 'qual é a literatura produzida sobre a distribuição médica no território brasileiro?'. Para solucionar a pergunta da pesquisa, foi realizada uma revisão integrativa da literatura com o objetivo de mapear a literatura disponível, a fim de fornecer um diagnóstico sobre a abordagem científica relacionada ao tema.

\section{Material e métodos}

A metodologia proposta para o estudo é uma revisão integrativa desenvolvida nos moldes das diretrizes do Joanna Briggs Institute $(\mathrm{JBI})^{10}$. O JBI é uma organização internacional sem fins lucrativos que compõe a School of Translational Science of Faculty of Health Science, University of Adelaide, na Austrália. No estudo em questão, foi aplicada a mnemônica População, Conceito e Contexto (PCC). Entre os contextos, População refere-se à população ou a um problema que pode ser um indivíduo ou um grupo em uma condição específica; Conceito podem ser todos os elementos detalhados e relevantes a serem considerados; Contexto é determinado segundo o objetivo e a pergunta da revisão, sendo definido pelos fatores culturais. Para a pergunta norteadora do presente estudo, foram definidas:

- População: médicos;

- Conceito: distribuição médica;

- Contexto: Brasil.

Quanto à estratégia de busca, foram consultadas seis bases de dados: Scientific 
Eletronic Library Online (SciELO), Literatura Latino-Americana e do Caribe em Ciências da Saúde (Lilacs), PubMed, Cochrane, Scopus e Web of Science. Para adequação das buscas, foram utilizados os descritores controlados e todos os seus respectivos descritores não controlados do Medical Subject Heading (MeSH) e do
Descritores em Ciências da Saúde (DeCS). Também foram considerados os termos booleanos junto aos descritores: AND, OR e NOT. Os descritores controlados utilizados pela busca e que serviram de referência para a seleção dos descritores não controlados, assim como as palavras-chave para cada item da mnemônica, estão descritos no quadro 1.

Quadro 1. Descritores e palavras-chave

\begin{tabular}{|c|c|c|c|}
\hline Estratégia PCC & Descritores controlados & Descritores não-controlados & Palavras-Chave \\
\hline \multirow[t]{2}{*}{ População (P) } & $\begin{array}{l}\text { Médicos OR "Cuidados } \\
\text { Médicos" }\end{array}$ & Médicos OR "Atendimento Médico" & \\
\hline & $\begin{array}{l}\text { Physicians OR "Medical } \\
\text { Care" }\end{array}$ & Physician & \\
\hline \multirow[t]{2}{*}{ Conceito (C) } & $\begin{array}{l}\text { "Distribuição de Médicos" } \\
\text { OR Demografia }\end{array}$ & $\begin{array}{l}\text { "Análise Demográfica" OR "Análise Transversal" OR "Método de Brass" OR } \\
\text { "Demógrafos" OR "Distribuição da População" OR "Distribuição Populacional" } \\
\text { OR "Inquéritos Demográficos" OR "Estudo Nacional da Despesa Familiar" OR } \\
\text { "Estudo Nacional da Despesa Familiar (ENDEF)" OR "ENDEF" OR "Inquéritos } \\
\text { Populacionais" OR "Levantamento Demográfico" OR "Levantamentos Demográ- } \\
\text { ficos" OR "Pesquisa Demográfica" OR "Pesquisas Demográficas" }\end{array}$ & $\begin{array}{l}\text { "Demografia } \\
\text { médica" }\end{array}$ \\
\hline & $\begin{array}{l}\text { "Physicians Distribution" } \\
\text { OR Demography }\end{array}$ & $\begin{array}{l}\text { Demographics OR "Reverse Survival Method" OR "Method, Reverse Survival" } \\
\text { OR "Methods, Reverse Survival" OR "Reverse Survival Methods" OR "Population } \\
\text { Spatial Distribution" OR "Distribution, Population Spatial" OR "Distributions, Po- } \\
\text { pulation Spatial" OR "Population Spatial Distributions" OR "Spatial Distribution, } \\
\text { Population" OR "Spatial Distributions, Population" OR "Brass Technique" OR } \\
\text { "Technique, Brass" OR "Brass Technic" OR "Technic, Brass" OR "Demographers" } \\
\text { OR "Demographer" "Demographic Accounting" OR "Accounting, Demographic" } \\
\text { OR "Demographic Analysis" OR "Analysis, Demographic" OR "Demographic } \\
\text { Analyses" OR "Analyses, Demographic" OR "Demographic and Health Surveys" } \\
\text { OR "Factors, Demographic" OR "Demographic Factors" OR "Factor, Demographic" } \\
\text { OR "Demographic Factor" OR "Family Reconstitution" OR "Family Reconstitu- } \\
\text { tions" OR "Reconstitution, Family" OR "Reconstitutions, Family" OR "Historical } \\
\text { Demography" OR "Demographies, Historical" OR "Historical Demographies" OR } \\
\text { "Demography, Historical" OR "Impacts, Demographic" OR "Demographic Im- } \\
\text { pacts" OR "Impact, Demographic" OR "Demographic Impact" OR "Multiregional } \\
\text { Analysis" OR "Analysis, Multiregional" OR "Multiregional Analyses" OR "Analy- } \\
\text { ses, Multiregional" OR "Period Analysis" OR "Analyses, Period" OR "Period Analy- } \\
\text { ses" OR "Analysis, Period" OR "Population Distribution" OR "Distribution, Popula- } \\
\text { tion" OR "Distributions, Population" OR "Population Distributions" OR "Prehistoric } \\
\text { Demography" OR "Demographies, Prehistoric" OR "Prehistoric Demographies" } \\
\text { OR "Demography, Prehistoric" OR "Stable Population Method" OR "Method, } \\
\text { Stable Population" OR "Methods, Stable Population" OR "Population Methods, } \\
\text { Stable" OR "Stable Population Methods" OR "Surveys, Demographic" OR "Demo- } \\
\text { graphic Surveys" OR "Survey, Demographic" OR Demographic Survey" }\end{array}$ & \\
\hline \multirow[t]{2}{*}{ Contexto (C) } & Brasil & & $\begin{array}{l}\text { Brasileiro OR } \\
\text { Brasileiros }\end{array}$ \\
\hline & Brazil & & $\begin{array}{l}\text { Brazilian OR } \\
\text { Brazilians }\end{array}$ \\
\hline
\end{tabular}

Fonte: Elaboração própria. 
O levantamento bibliográfico foi realizado por pares de pesquisadores durante o mês de setembro de 2020 com os critérios de elegibilidade: artigos completos disponíveis; artigos disponíveis nos idiomas português, inglês ou espanhol; artigos completos que respondessem à pergunta norteadora do estudo; artigos completos que trataram o território em âmbito federal. Para auxiliar a leitura exaustiva dos títulos, resumos e conteúdos, foi utilizado o software Start. Com relação ao recorte temporal, optou-se por utilizar todo o período disponível nas bases para obtenção de uma quantidade maior de trabalhos.

A busca inicial resultou em um total de 491 trabalhos. Após a aplicação dos critérios de elegibilidade, a amostra final foi composta de 10 estudos. O processo de seleção dos estudos e o resultado final da revisão encontram-se descritos na figura 1.

Figura 1. Resultado final da revisão

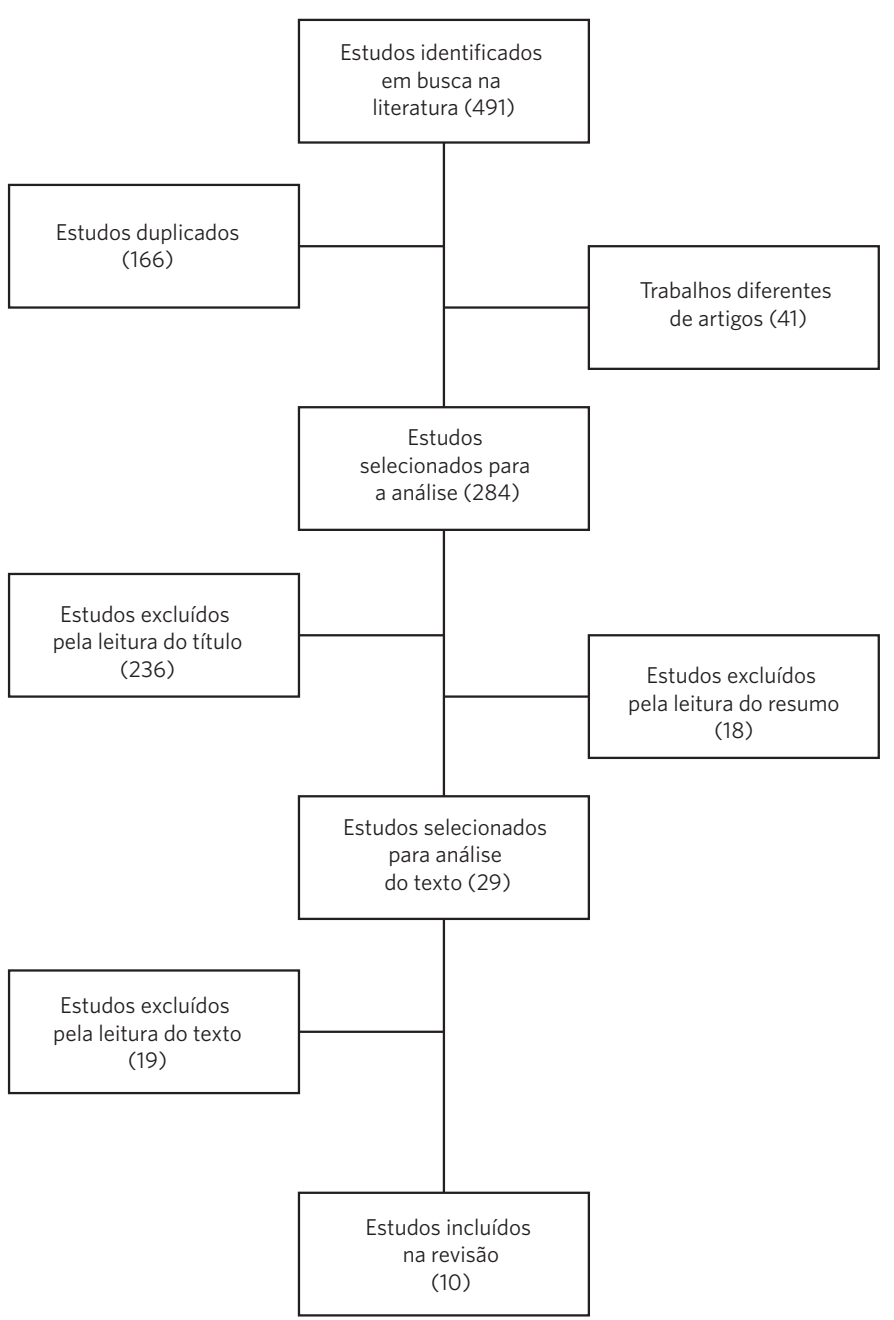




\section{Resultados}

\section{O processo de busca finalizou com a seleção de 10 artigos científicos relacionados à}

distribuição médica no território brasileiro. No quadro 2, é possível identificar os autores, os títulos, os objetivos e os anos de publicação dos trabalhos.

Quadro 2. Resumo dos artigos

\begin{tabular}{|c|c|c|c|}
\hline Autor & Título & Objetivo & Ano \\
\hline Povoa e Andrade ${ }^{\mathbf{1 1}}$ & $\begin{array}{l}\text { Distribuição geográfica dos } \\
\text { médicos no Brasil: uma aná- } \\
\text { lise a partir de um modelo de } \\
\text { escolha locacional. }\end{array}$ & $\begin{array}{l}\text { Modelar a decisão locacional dos médicos com base nos dados da } \\
\text { PNAD e analisar o papel de alguns fatores destacados na literatura } \\
\text { como sendo importantes para a compreensão de sua escolha locacio- } \\
\text { nal e, consequentemente, da sua distribuição geográfica no Brasil. }\end{array}$ & 2006 \\
\hline $\begin{array}{l}\text { Martins Sant'anna, Rocha e } \\
\text { Vieira } \mathbf{1 2}\end{array}$ & $\begin{array}{l}\text { Programa Mais Médicos: Uma } \\
\text { Revisão Integrativa. }\end{array}$ & $\begin{array}{l}\text { Descrever o conhecimento produzido na literatura acerca da criação do } \\
\text { Programa Mais Médicos e sua repercussão no Brasil. }\end{array}$ & 2017 \\
\hline $\begin{array}{l}\text { Stralen, Massote, Carvalho e } \\
\text { Girardi13 }\end{array}$ & $\begin{array}{l}\text { Percepção de médicos sobre } \\
\text { fatores de atração e fixação } \\
\text { em áreas remotas e desassisti- } \\
\text { das: rotas da escassez. }\end{array}$ & $\begin{array}{l}\text { Identificar os fatores que contribuem para atrair e reter médicos em } \\
\text { áreas remotas e desassistidas do País. }\end{array}$ & 2017 \\
\hline Martinez $^{\mathbf{1 4}}$ & $\begin{array}{l}\text { A abordagem equitativa de } \\
\text { gênero como uma estratégia } \\
\text { de gestão para fixação de mé- } \\
\text { dicos em áreas vulneráveis. }\end{array}$ & $\begin{array}{l}\text { Debater sobre estratégias de gestão em saúde capazes de atrair e reter } \\
\text { profissionais médicos para áreas mais vulneráveis do País. }\end{array}$ & 2017 \\
\hline Weber ${ }^{15}$ & $\begin{array}{l}\text { Dialectics of a medical provi- } \\
\text { sion policy in priority areas in } \\
\text { Brazil. }\end{array}$ & $\begin{array}{l}\text { Problematizar o Programa Mais Médicos, com o propósito de contri- } \\
\text { buir para o debate sobre as políticas de provimento médico no Brasil. }\end{array}$ & 2017 \\
\hline $\begin{array}{l}\text { Oliveira, Gabriel, Dal Poz e } \\
\text { Dussault5 }\end{array}$ & $\begin{array}{l}\text { Challenges for ensuring availa- } \\
\text { bility and accessibility to heal- } \\
\text { th care services under Brazil's } \\
\text { Unified Health System (SUS). }\end{array}$ & $\begin{array}{l}\text { Compreender os desafios dos formuladores de políticas e gestores do } \\
\text { SUS para garantir a disponibilidade e a acessibilidade geográfica aos } \\
\text { prestadores de serviços de saúde. }\end{array}$ & 2017 \\
\hline Ezequiel et al. ${ }^{\mathbf{1 6}}$ & $\begin{array}{l}\text { Geographical distribution of } \\
\text { medical graduates from a } \\
\text { public university. }\end{array}$ & $\begin{array}{l}\text { Avaliar a distribuição geográfica e a trajetória de carreira dos gradua- } \\
\text { dos em medicina e os fatores associados à escolha do local da prática. }\end{array}$ & 2017 \\
\hline Silva et al. ${ }^{\mathbf{1 7}}$ & $\begin{array}{l}\text { Cost of providing doctors in } \\
\text { remote and vulnerable areas: } \\
\text { Programa Mais Médicos in } \\
\text { Brazil. }\end{array}$ & $\begin{array}{l}\text { Avaliar o Programa Mais Médicos (PMM) no Brasil estimando o au- } \\
\text { mento proporcional do número de médicos nos municípios participan- } \\
\text { tes e os custos do programa, estratificado pelo componente de custo e } \\
\text { fonte de financiamento. }\end{array}$ & 2018 \\
\hline $\begin{array}{l}\text { Figueiredo, Mckinley, Lima e } \\
\text { Azevedo } \mathbf{1 8}\end{array}$ & $\begin{array}{l}\text { Medical school expansion } \\
\text { policies: educational access } \\
\text { and physician distribution. }\end{array}$ & $\begin{array}{l}\text { Analisar o impacto das políticas de expansão das escolas médicas so- } \\
\text { bre as desigualdades regionais na distribuição do tamanho das turmas } \\
\text { de graduação e a capacidade de atrair e reter médicos e de expandir as } \\
\text { unidades de saúde. }\end{array}$ & 2019 \\
\hline $\begin{array}{l}\text { Pinto Júnior, Amorim e Aqui- } \\
\text { no }\end{array}$ & $\begin{array}{l}\text { Programa Mais Médicos: con- } \\
\text { texto de implantação e efeito } \\
\text { no provimento de médicos na } \\
\text { atenção primária à saúde no } \\
\text { Brasil, } 2008 \text { a } 2016 .\end{array}$ & $\begin{array}{l}\text { Caracterizar os municípios brasileiros que compunham os perfis priori- } \\
\text { tários para implantação do Programa Mais Médicos (PMM) e avaliar o } \\
\text { efeito do programa no provimento emergencial de médicos na Atenção } \\
\text { Primária à Saúde (APS) no Brasil, segundo contexto de implantação. }\end{array}$ & 2020 \\
\hline
\end{tabular}


O estudo de Povoa e Andrade utilizou dados da Pesquisa Nacional por Amostra de Domicílios (PNAD) para determinar a decisão locacional dos médicos e analisar fatores importantes para sua distribuição no território ${ }^{11}$. De acordo com os autores, a literatura aponta que as oportunidades de trabalho para o cônjuge e o local onde o médico recebeu seu treinamento exercem forte influência na escolha locacional'11. Os autores destacam evidências internacionais, apontando que os médicos tendem a permanecer no local onde a residência médica foi realizada, reforçando a importância da distribuição geográfica dos cursos de medicina e dos programas de residência para a distribuição equitativa de profissionais"1.

Os resultados do artigo apontam que as vagas nos programas de residência são fundamentais para atrair médicos para uma determinada região, sendo que a probabilidade de um médico ser não natural é maior em estados onde as vagas de residência são mais abundantes $\mathbf{1 1}^{11}$ Portanto, os autores indicam que a concentração de programas de residência nas regiões Sul e Sudeste são um dos fatores que promovem a distribuição desigual de médicos pelo território nacional1". Ademais, segundo os resultados, médicos mais jovens são mais propensos a mudar de localidade, e a probabilidade de um médico ser não natural é mais elevada em estados com maiores PIB per capita, indicando atração exercida pelo fator econômico"1.

$\mathrm{O}$ artigo desenvolvido por Martins et al. buscou realizar uma revisão integrativa da literatura sobre o PMM e sua repercussão no Brasil12. Segundo os autores, a concentração dos médicos brasileiros em grandes centros urbanos e em regiões mais desenvolvidas é histórica, acarretando problemas para fixar e prover tais profissionais em outras regiões do País ${ }^{12}$. Os habitantes das capitais possuem, em média, duas vezes mais médicos do que os de outras localidades do mesmo estado, com vários fatores influenciando a disparidade: indicadores sociais; condições de trabalho; precarização do vínculo trabalhista; contratos de trabalho temporários; disponibilidade de vagas nas faculdades; e custos elevados dos cursos privados ${ }^{\mathbf{1 2}}$.

Até o surgimento do PMM, várias iniciativas propostas pelo governo foram desenvolvidas para enfrentar a má distribuição médica: implementação do Sistema Único de Saúde (SUS), Projeto Rondon, Programa de Interiorização das Ações de Saúde e Saneamento (Piass), o Pisus, o Programa Saúde da Família (PSF), o Programa de Interiorização do Trabalho (Pits), o Fundo de Financiamento ao Estudante do Ensino Superior (Fies) e o Programa de Valorização do Profissional da Atenção Básica (Provab), com outros movimentos emergindo em consequência dos problemas ocasionados pela falta de médicos como o 'Cadê o Médico', desenvolvido pela Frente Nacional de Prefeitos, em 2013, com o objetivo de reivindicar a presença desses profissionais nos locais não atendidos ${ }^{12}$. Os autores destacam que o programa foi criado com o objetivo de ampliar o acesso e atenuar as desigualdades em saúde com a distribuição de médicos em municípios prioritários, entretanto, embora a maioria dos achados da revisão tenha sido favorável à criação da iniciativa, ela não resolve o problema da distribuição se tratar de uma questão gerada pela má administração de recursos, da qual os médicos também são vítimas $^{\mathbf{1 2}}$. Portanto, o programa criou polêmicas e conflitos ideológicos entre as classes médicas, motivando a construção de percepções baseadas em interesses diversos ${ }^{\mathbf{1 2}}$.

O trabalho de Stralen et al. ${ }^{13}$ buscou identificar os fatores que contribuem para fixação e retenção de médicos em áreas remotas. A má distribuição de médicos é reflexo das desigualdades endêmicas brasileiras, pois tais profissionais preferem regiões economicamente mais desenvolvidas por oferecerem maiores oportunidades profissionais, educacionais e de lazer ${ }^{13}$. Ademais, gestores municipais fazem uso de políticas de incentivos para atrair médicos, como aumento de salários e flexibilização da jornada de traba$1 h 0^{13}$. Entretanto, apesar do salário ter sido 
um atributo mencionado por quase todos os entrevistados, a combinação de incentivos financeiros e não financeiros é importante para atração e fixação de profissionais ${ }^{\mathbf{1 3}}$.

O estudo de Martinez abordou a equidade de gênero como uma estratégia de gestão para fixação de médicos em áreas vulneráveis ${ }^{\mathbf{1 4}}$. Segundo a autora, a utilização de mulheres nas áreas vulneráveis é uma abordagem viável porque as mulheres são mais leais às organizações e costumam favorecer a retenção masculina, impactando na qualidade da assistência e nos indicadores de saúde ${ }^{14}$. A autora pontua duas abordagens estratégicas para fixação: governamental e organização $0^{\mathbf{1 4}}$.

Como abordagens governamentais, Martinez indica: contratos laborais em tempo parcial para equilibrar as atividades profissionais com as demandas familiares; programas municipais que favorecem o emprego do cônjuge; disponibilidade em creches ou escolas públicas de qualidade; políticas municipais de desenvolvimento de cultura, esporte e lazer ${ }^{14}$. Como abordagens organizacionais, Martinez cita: manutenção de clima organizacional favorável; desenvolvimento de políticas internas que valorizem a família com flexibilidade de horários; desenvolvimento de programas de saúde com foco no sexo feminino; e programas voltados para mapeamento e desenvolvimento de talentos ${ }^{\mathbf{1 4}}$.

O trabalho de Weber ${ }^{15}$ problematiza o PMM para discutir as políticas de provimento médico no Brasil. Para os autores, a garantia constitucional do acesso universal à saúde encontra barreiras por conta da dimensão territorial brasileira e de um sistema de transporte limitado, com precariedade das malhas de mobilidade, destacando o caráter periférico de várias regiões ${ }^{15}$. Tal falta de infraestrutura básica local e regional dificulta a diversidade econômica e cultural, sendo menos convidativa para médicos viverem nesses lugares ${ }^{15}$.

A fim de combater o problema da distribuição de médicos, o governo criou o PMM em 2013, causando grande reação nas entidades médicas por conta a figura do intercambista (médico formado em instituição estrangeira), sem a necessidade de revalidação do diploma, pois, sem a devida comprovação pelas universidades brasileiras do conhecimento mínimo exigido no Brasil para a prática médica, a saúde da população assistida por tais profissionais poderia ser posta em risco ${ }^{15}$. Ademais, a proficiência na língua portuguesa é necessária para uma comunicação adequada entre médico e paciente que assegure o pleno entendimento entre os interlocutores ${ }^{15}$. Para concluir, o autor menciona que, para melhorar a saúde no País, $o$ SUS precisa de outros requisitos além da figura do médico, o qual se torna inútil se não houver condições seguras e éticas para se chegar a um diagnóstico, para estabelecer o plano terapêutico e monitorar a reabilitação do paciente ${ }^{15}$.

O artigo de Oliveira et al. ${ }^{5}$ buscou compreender os desafios dos formuladores de políticas públicas e gestores do SUS para garantir a disponibilidade e a acessibilidade geográfica aos prestadores de serviços de saúde. Segundo os autores, o desequilíbrio é causado por vários fatores, que podem ser divididos em: individual; organizacional; e relacionados aos sistemas (saúde, educação, institucional) e às características dos municípios, incluindo o ambiente econômico, sociocultural, histórico e políti$\mathrm{co}^{5}$. Nos estudos encontrados pelos autores, as principais causas de distribuição inadequada foram: características dos municípios, incluindo o Produto Interno Bruto (PIB), o Índice de Desenvolvimento Humano, os níveis de vulnerabilidade social, as condições de violência e as oportunidades de trabalho; características individuais, por exemplo, idade, oportunidades de trabalho para cônjuges, origem em ambientes urbanos e renda familiar, características do sistema de ensino, existência de curso médico, residência médica e possibilidades de educação continuada; e, finalmente, características organizacionais, incluindo remuneração, condições de trabalho, plano de carreira e reconhecimento profissional ${ }^{5}$.

Em 2003, o Ministério da Saúde criou o Departamento de Gestão de Trabalho e Educação em Saúde (SGTES), responsável por formular políticas de gestão do trabalho em 
saúde, além da capacitação e qualificação de recursos humanos em saúde, regulamentação profissional e descentralização da gestão do trabalho e da educação nos estados do Brasil 5 . No entanto, vê-se que falta algo na formulação de uma política clara e de longo prazo para governar os recursos humanos em saúde no Brasil, embora várias estratégias específicas e limitadas tenham sido identificadas para enfrentar esses desafios ${ }^{5}$. As análises dos autores mostram que não foram encontradas iniciativas de políticas nas áreas de regulação do setor privado, mostrando que há necessidade de iniciativas e políticas que considerem todas as facetas do mercado de trabalho em saúde no Brasil, como, por exemplo, a coexistência entre o setor público e o privado 5 .

O estudo de Ezequiel et al. ${ }^{16}$ avaliou a distribuição geográfica e a trajetória de carreira de 563 egressos do curso de medicina da Universidade Federal de Juiz de Fora, que responderam ao questionário aplicado. A análise das respostas revelou que as razões para a escolha de trabalhar em cidades menores incluíam se havia residência de membros da família, percepção de que a localização tinha oportunidades educacionais, trabalho em unidade de atenção primária à saúde e recebimento de convites durante a graduação ${ }^{16}$. Para aqueles que escolheram cidades maiores, os motivos eram que o local apresentava condições favoráveis de recreação e entretenimento, se receberam convite durante o treinamento de residência e no último ano de treinamento médico ${ }^{16}$.

O trabalho de Silva et al. ${ }^{17}$ estimou o aumento proporcional do número de médicos nos municípios participantes do PMM e dos custos do programa. O programa promoveu um adicional de 14.462 médicos para áreas remotas e vulneráveis em 3.785 municípios e 34 distritos sanitários especiais indígenas a um custo anual estimado em US\$1,1 bilhão, que cobriu provisões médicas, educação continuada e supervisão/mentoria'17. Os autores consideram moderados os custos do programa em relação aos benefícios em potencial à saúde da população ${ }^{17}$.
O estudo de Figueiredo et al. ${ }^{18}$ utilizou uma estimativa para averiguar como a expansão do ensino de medicina pode contribuir para reduzir os problemas da má distribuição médica no Brasil. Os resultados do estudo sugerem que municípios com novas escolas médicas apresentaram aumento na proporção de médicos por mil habitantes e na relação de estabelecimentos de saúde por habitante, demonstrando potencial para atrair e reter médicos, bem como fortalecer a infraestrutura de saúde. Os municípios mais distantes das capitais apresentaram maior aumento na razão médico por habitante ${ }^{18}$.

Os autores argumentam que países com escassez de força de trabalho em saúde e desigualdades poderiam utilizar a estratégia de expansão da oferta de cursos de medicina como forma de combate ao problema ${ }^{\mathbf{1 8}}$. Segundo os autores, os resultados iniciais no Brasil mostraram que tais estratégias poderiam fortalecer as redes de serviços em áreas carentes, apoiando a implementação da Cobertura Universal de Saúde18.

Por fim, o artigo de Pinto Júnior et al. ${ }^{19}$ buscou caracterizar os municípios brasileiros que compunham os perfis prioritários para implementação do PMM. Os resultados indicam que o programa auxiliou no incremento de profissionais na atenção primária em municípios com mais de $20 \%$ dos habitantes vivendo em situação de pobreza ${ }^{19}$. Portanto, a política do PMM foi exitosa no provimento emergencial para fortalecimento da atenção básica e do SUS ${ }^{19}$.

\section{Discussão}

A análise dos resultados apresenta uma disparidade entre o interesse científico com o tempo de existência do problema. O Decreto $n^{0} 53.642$, de 1964, já buscava soluções para a má distribuição médica, e, ao logo dos anos, diversas iniciativas foram desenvolvidas 5,7 . Entretanto, dos artigos selecionados para a amostra final, nove foram desenvolvidos a 
partir do ano de 2017, indicando uma possível influência do PMM para o repentino aumento de interesse científico no assunto, pois tal iniciativa criou polêmicas e conflitos na comunidade médica ${ }^{\mathbf{2}}$.

Com relação aos motivos para a má distribuição médica, os resultados apresentaram vários fatores que vão além das questões pecuniárias. Os médicos procuram regiões com melhores desenvolvimentos econômicos e sociais, com melhores escolas para filhos, oportunidades de trabalho para o cônjuge e de lazer6,13,14. Outro fator associado é o local onde o médico recebeu sua formação, portanto, disponibilidade de vagas nas faculdades, custos elevados dos cursos privados e a concentração de programas de residência nas regiões Sul e Sudeste promovem a distribuição desigual11,12.

Ademais, elementos organizacionais também influenciam a escolha do profissional pelo local de trabalho ${ }^{\mathbf{1 4}}$. Weber ${ }^{\mathbf{1 5}}$ destaca que o SUS precisa tornar as mais diversas regiões mais convidativas aos médicos provendo condições seguras e éticas para melhorar a resolução diagnóstica e as opções terapêuticas. As condições de trabalho e a precarização do vínculo trabalhista criam situações de insegurança laboral, que podem afugentar os médicos de áreas menos desenvolvidas ${ }^{5,12}$.

A falta de estrutura organizacional pode levar a erros médicos com potencial de culminar em processos judiciais. De acordo com Guest et al. ${ }^{20}$, processos judiciais são classificados pelos médicos como uma fonte de tensão equivalente às preocupações financeiras, aos problemas domiciliares e frustrações com a morte de pacientes. Portanto, é compreensível a busca por parte dos médicos de locais com maior estrutura, e é evidente que o problema vai além da falta de médicos ${ }^{15}$.

$\mathrm{O}$ artigo de Weber pontua a precariedade das malhas de mobilidade e a má distribuição de médicos como um reflexo das disparidades econômicas e sociais no território brasileiro ${ }^{15}$. É notória no Brasil a presença de cidades com indicadores sociais e econômicos compatíveis com os de países desenvolvidos, contrastando com municípios em condições análogas às regiões africanas de extrema pobreza. Regiões menos desenvolvidas dificilmente poderão propiciar boas condições de trabalho para cônjuges, boas escolas para os filhos e opções mínimas de lazer e entretenimento.

Portanto, o problema da má distribuição de médicos é mais complexo e de difícil solução, pois está intimamente ligado às diferenças sociais e econômicas que o Brasil apresenta. Para solucionar o problema da má distribuição de médicos, o Brasil precisaria resolver primeiro as diferenças sociais e econômicas das suas regiões. Porém, para conseguir atingir seus objetivos de igualdade econômica e social, o Brasil precisa de uma distribuição mais equitativa da força de trabalho em saúde, resultando em um ciclo sem solução?

O governo busca solucionar o problema com a expansão da oferta de cursos de medicina pela política do PMM. O aumento excessivo de médicos no Brasil, privilegiando a quantidade ao invés da qualidade não garante a fixação dos profissionais em áreas do interior do País, pois, além da formação, o profissional precisa receber capacitação e ter garantias trabalhistas e estruturais nos locais de atuação²1. A perspectiva com a atual expansão do ensino é um aumento substancial na quantidade de profissionais médicos no Brasil, como pode ser observado pela tabela 1 . 
Tabela 1. Projeção da oferta de médicos no Brasil

\begin{tabular}{lrr}
\hline Década & População & Médicos \\
\hline 1910 & 23.414 .177 & 13.270 \\
1920 & 30.635 .605 & 14.031 \\
$1930^{(1)}$ & -11236315 & 15.899 \\
1940 & 51944397 & 20.745 \\
1950 & 70.070 .457 & 26.120 \\
1960 & 93.139 .037 & 34.792 \\
1970 & 119.002 .706 & 58.994 \\
1980 & 146.002 .706 & 137.347 \\
$1991^{(2)}$ & 169.799 .926 & 219.084 \\
2000 & 190.732 .694 & 291.926 \\
2010 & 211.755 .692 & 364.757 \\
$2020(3)$ & $223.126 .917(4)$ & 498.395 \\
2030 & & $884.738(5)$ \\
\hline
\end{tabular}

Fonte: IBGE Censos Demográficos e CFM/Cremesp ${ }^{\mathbf{2 2}, 23}$. Demografia Médica ${ }^{\mathbf{2 4}}$

(1) No ano de 1930, por motivos políticos, o Censo não foi realizado. (2) Os Dados da População correspondem ao Censo que, por problemas técnicos, foi realizado em 1991. (3) Dados de médicos inscritos no CFM até 19/09/2020. (4) Projeção do IBGE para 203025 (5) Projeção do número de médicos formandos para 2030, considerando as atuais 29.987 vagas de ingresso nos cursos de medicina até 2025, considerando 36.780 a partir de 2026 quando todos os cursos iniciados até 2020 estarão formando as primeiras turmas, sem considerar a abertura de novos cursos e/ou aumentos de vagas dos cursos preexistentes e sem considerar os óbitos de médicos no período entre 2020 e $2030^{26}$

Os dados de crescimento populacional brasileiro e a quantidade de médicos no País entre 1980 e 2020 possibilitam a projeção das taxas de crescimento até 2030. Com os dados disponíveis, mantendo-se os parâmetros atuais até 2025 e estimando aberturas a partir de 2026 com a quantidade de cursos iniciados em 2020, é possível projetar uma proporção de médicos para cada mil habitantes de 3,8 em 2030, o que representará um crescimento de $71 \%$ com relação à década de 2020. A relação das taxas de crescimento da população brasileira, projetados podem ser vistos no gráfico 1 . 
Gráfico 1. Relação das taxas de crescimento da população brasileira, população de médicos e da razão do número de médicos para mil habitantes entre 1980 e 2020 com as projeções para 2030

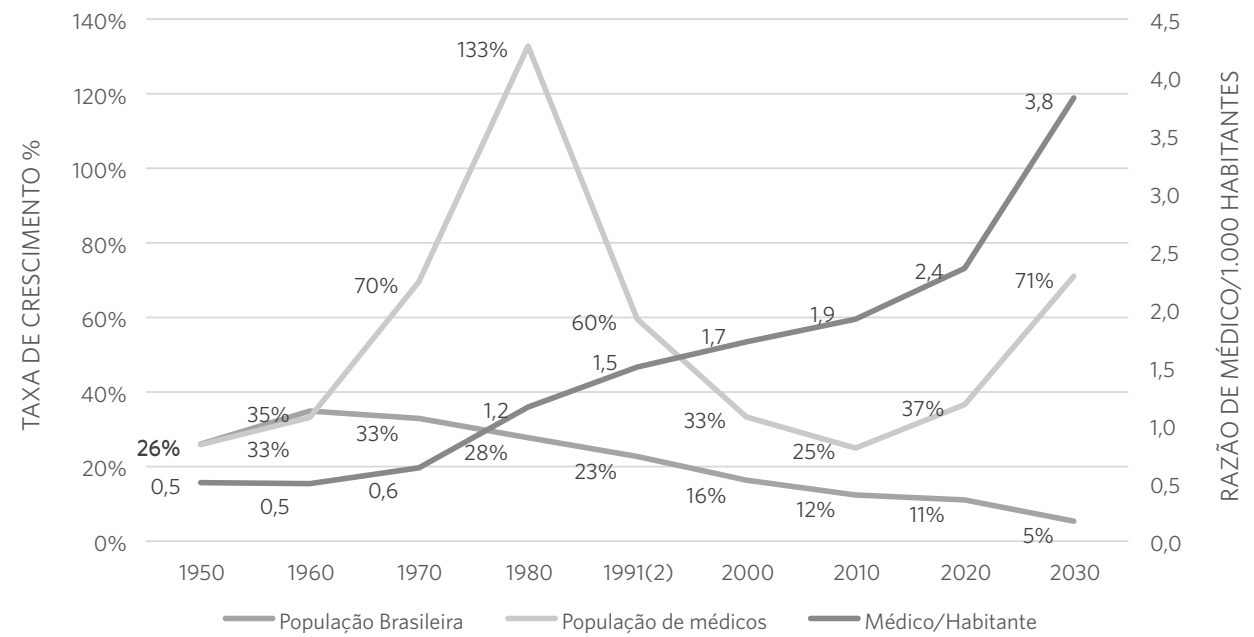

Fonte: Elaborado com dados do IBGE22 e Demografia Médica4.

O pico do crescimento da população de médicos ocorrido na década de 1980 é reflexo das políticas educacionais do final dos anos 1960. Em 1968, ocorreu a Reforma Universitária, promovendo uma forte expansão do ensino superior brasileiro como um todo, incluindo a abertura de novos cursos de graduação em medicina ${ }^{27}$. Entre 1968 e 1989, foram abertos 34 novos cursos de medicina, uma expansão de 47 para 81 cursos de graduação de medicina oferecidos no Brasil28. A expansão do ensino superior de medicina após a Reforma Universitária ocorreu majoritariamente pela via privada, podendo ser observado pela proporção de vagas de cursos privados frente aos públicos, que chegarão a $75 \%$ do total de vagas quando todos os cursos estiverem formando profissionais ${ }^{26}$.

Pesquisa da Organização para a Cooperação e Desenvolvimento Econômico (OCDE) comparando 40 países chegou à média de 3,2 médicos por mil habitantes, com a média brasileira de 1,8 médico por mil habitantes ${ }^{29}$. A mesma pesquisa, em 2019, mostrou uma média de 3,5 médicos por mil habitantes, tendo o Brasil a média de 1,8 médico por mil habitantes. Os países com a maior média foram a Grécia (6,1 médicos por mil habitantes), seguida por Áustria $(5,2)$; Portugal $(5,0)$; Noruega $(4,7)$; Lituânia $(4,6)$; Suíça e Alemanha $(4,3)$; Suécia $(4,1)$; Rússia, Dinamarca e Itália $(4,0)$; Espanha e Islândia (3,9); República Tcheca e Austrália (3,7); e Países Baixos (3,6). Os países com média abaixo de 1 médico por 1000 habitantes foram África do Sul $(0,8)$, Índia $(0,8)$ e Indonésia $(0,3)^{30}$.

Países que possuem dimensão territorial semelhante à do Brasil, como EUA, China e Índia, possuem, respectivamente, as médias de 2,6; 2,0 e 0,8 médicos por mil habitantes ${ }^{30}$. Entretanto, ao se compararem as populações estimadas, enquanto o Brasil possui 211 milhões de habitantes em 2020, a população chinesa é de 1.439 bilhões, a indiana de 1.380 bilhões e a estadunidense de 331 milhões ${ }^{31,32}$. Com populações maiores que a brasileira, é notável que a quantidade de médicos formados seja maior. No entanto, ao comparar a quantidade 
de cursos de graduação em medicina, há um descompasso com as proporções médicas apontadas pela OCDE.

Com relação ao número de cursos de medicina, com exceção da Índia, que possui 457 cursos, o Brasil ocupa o segundo lugar, com 342 cursos de medicina, à frente da China, com 160 cursos, e dos EUA, com 19528,33.

Com a grande expansão do número de cursos de medicina a partir do PMM, em 2013, mantendo o atual número de 342 escolas e 35.388 vagas para o primeiro ano até 2025 e 36.789 vagas para o primeiro ano a partir de 2026, com a previsão do número de médicos formados no Brasil em 2030, o País dará um salto em menos de 20 anos da razão de 1,8 para cerca de 3,9 médicos para 1000 habitantes. Não é escopo deste artigo abordar os vários aspectos da rapidez do crescimento da taxa de médicos em função da capacitação técnica e pedagógica docente e de preceptores para supervisionarem essa quantidade abrupta de graduandos, assim como a infraestrutura da rede de saúde para transformar a atual realidade da má qualidade da assistência prestada à população, porém, uma aparente vantagem para o SUS pode vir a ser uma grande catástrofe pessoal e profissional com um mercado de trabalho que pode ter uma enorme oferta de mão de obra pouco qualificada.

Em estudo sobre a distribuição geográfica da expansão de cursos médicos, notou-se uma expansão dos cursos privados nas regiões Nordeste, Sudeste e Sul, e cursos públicos na região Centro-Oeste e Norte. A análise regional mais detalhada mostrou que a política pública tem fracassado em seu objetivo de combater a má distribuição médica no território nacional por meio da abertura dos novos cursos médicos, pois mantém a concentração em estados do Sul e do Sudeste ${ }^{28}$.

Outro ponto de destaque nos resultados é a ausência de trabalhos científicos relacionados aos hospitais-escola. Um hospital-escola congrega elementos de um hospital tradicional com a prática do ensino, possuindo, em suas instalações, as presenças de estudantes e residentes ${ }^{34}$. A distribuição geográfica dos hospitais-escola no Brasil acompanha as áreas com maior densidade de médicos, sendo notória a concentração dos hospitais-escola nas regiões Sul e Sudeste, onde também está presente a maior concentração de médicos e de programas de residência segundo Povoa e Andrade"1.

Um hospital tradicional apresenta características que o diferem dos demais tipos organizacionais existentes por conta da atuação de uma equipe multidisciplinar que presta desde assistência à saúde ao atendimento ambulatorial e internações, mediante a utilização de tecnologias leves (trabalho vivo) e duras (equipamentos) ${ }^{35}$. Os hospitais-escola, além das características dos hospitais tradicionais, são importantes para a região onde estão localizados porque fornecem bens públicos sofisticados e especializados e ajudam na difusão do conhecimento em decorrência da pesquisa e do ensino ${ }^{36}$.

Desde a promulgação do Decreto $n^{\circ} 63.341$, de $1^{\circ}$ de outubro de 1968 , a obrigatoriedade de um curso de medicina de ter um hospital-escola deixou de existir ${ }^{37}$. As instituições são incentivadas a realizar convênios com organizações médicas para que os alunos possam ter o ensino prático. Entretanto, os convênios realizados têm sido insuficientes para atender à exigência do Ministério da Educação (MEC) de pelo menos cinco leitos por estudante, afetando a formação do médico e o atendimento ${ }^{38}$.

Além da quantidade mínima de leitos hospitalares, o Conselho Federal de Medicina (CFM) também considera outros dois critérios como sendo necessários para um processo de ensino e aprendizagem minimante qualificado. São eles: acompanhamento da equipe da Estratégia Saúde da Família (ESF) por no máximo três alunos e presença de hospital com mais de 100 leitos $^{38}$. Os critérios adotados pelo CFM não são arbitrários, mas baseados nos itens das portarias presentes nos editais que regulamentaram as aberturas das escolas médicas no Brasil a partir do PMM, os quais foram flexibilizados em 2015, tornando os 
critérios mais subjetivos ${ }^{39}$. De acordo com o Radiografia Médica, das 163 escolas abertas entre 2011 e 2019, 39,9\% (65 escolas) estão em cidades que descumprem todos os requisitos mínimos anteriormente estipulados, 41,1\% (67 escolas) apresentam irregularidades em dois parâmetros e 13,5\% (22 escolas) apresentam um parâmetro irregular (CFM, 2020). Apenas $5,5 \%$ (9 escolas) atenderam a todos os requisitos do $\mathrm{CFM}^{39}$.

A análise do CFM também envolveu todas as 342 escolas abertas até 2019 , e o resultado, segundo a instituição, é preocupante, pois 26,6\% (91 escolas) estão em 65 municípios que não atendem aos padrões mínimos de ensino e aprendizagem estabelecidos ${ }^{39}$. Outros $38 \%$ (130 escolas) estão em cidades que cumprem apenas um requisito e 19\% (66 escolas) estão em municípios que atendem a dois parâme$\operatorname{tros}^{39}$. Apenas a minoria, $16,1 \%$ (55 escolas), está localizada em municípios que atendem a todos os requisitos estabelecidos ${ }^{39}$.

A informação do CFM preocupa, pois o local de formação do médico, principalmente na residência médica, é essencial para a retenção dos profissionais por exercerem forte influência na escolha do local de trabalho, ${ }^{5,11,14}$. A presença de um hospital-escola atrai médicos residentes para uma determinada localidade, aumentando as chances de o profissional permanecer naquela região, criando postos de trabalho que precisam ser ocupados, aumentando as taxas de atração e retenção dos profissionais da saúde. Portanto, torna-se necessário investigar mais profundamente a relação dos hospitais-escola com a distribuição médica pelo território nacional.

Sem a devida estrutura para a retenção do profissional recém-formado no local da graduação, a abertura de novos cursos de graduação em medicina incentivada pelo PMM é inócua. Se a expectativa governamental é forçar os médicos a buscarem empregos em áreas distantes pela falta de oportunidades nos grandes centros, a medida poderá levar anos para surtir o efeito desejado, mantendo a população vulnerável com acesso restrito à saúde. Entretanto, o próprio PMM apresentou uma proposta que trouxe resultados satisfatórios ao acesso à saúde da população, presente no segundo artigo da lei: alocação direta da força de trabalho ${ }^{8}$.

A alocação direta de profissionais médicos nas regiões de alta vulnerabilidade social atingiu os objetivos do PMM, com boa avaliação nas Unidades Básicas de Saúde (UBS) contempladas por ele e com melhora nos indicadores de saúde locais ${ }^{40,41}$. O PMM é bem avaliado nas UBS, e a nacionalidade do médico, cubano ou brasileiro, não altera a satisfação do paciente com o atendimento ${ }^{\mathbf{4 1}, 42}$. Entretanto, com a nova política governamental que resultou na saída dos médicos intercambistas, ocorreu um desabastecimento nos postos de trabalho, impactando milhares de pessoas em áreas vulneráveis ${ }^{43}$. As vagas deixadas pelos médicos intercambistas foram preenchidas por brasileiros, mas a alta desistência provocou desabastecimento de força de trabalho médica em várias cidades ${ }^{\mathbf{4 4}}$.

Há, portanto, interesse de médicos brasileiros de fazerem parte do PMM. Cabe ao Governo Federal criar incentivos para a fixação do profissional médico em áreas vulneráveis durante todo o período estipulado. Uma das ações governamentais para a fixação profissional seria o investimento na estrutura das UBS, pois muitas possuem qualidade questionável ${ }^{45}$. Outra ação sugerida, pelo fato de o PMM também contemplar o ensino médico, seria uma mudança curricular voltada para a Atenção Primária à Saúde (APS), pois, atualmente, há um conflito entre o ensino e a realidade nacional, que dificulta a fixação médica em áreas vulneráveis ${ }^{46}$. Por fim, ações afirmativas poderiam ser utilizadas em áreas vulneráveis para a inclusão de alunos locais nas faculdades de medicina como o Argumento de Inclusão Regional (AIR), que consiste em um aumento na nota de um aluno dependendo da localização original do estudante, evitando a ocupação demasiada das vagas de graduação por alunos forasteiros que irão voltar para suas regiões de origem após a conclusão do curso de graduação ${ }^{47}$. 


\section{Conclusões}

A revisão integrativa da literatura sobre a distribuição médica no território brasileiro indica fatores pecuniários e não pecuniários como motivos para a má distribuição de médicos no Brasil: oportunidades de trabalho para cônjuges, disponibilidade de escolas para filhos, idade dos profissionais, localidade da formação e dos programas de residência e disponibilidade de opções de lazer. Com a grande expansão do número de cursos de medicina e das vagas de graduação a partir do PMM (2013), mantendo-se o atual número de cursos médicos, cuja distribuição mais uma vez concentrou-se nas regiões Sudeste e Sul, a projeção para 2030 é o aumento de $71 \%$ do número de médicos formados e da razão de médicos por mil habitantes de 1,8 para 3,8 , que pode ser comparada com a média entre os demais países membros da OCDE. Apesar das dificuldades da capacitação técnica e pedagógica docente e de preceptores para supervisionarem essa quantidade abrupta de graduandos, assim como da infraestrutura da rede de saúde para oferecer suporte ao ensino, há uma oportunidade para transformar as lacunas e a qualidade atual do acesso e da assistência prestada à população no SUS.

O grande trunfo do PMM frente aos demais programas de combate à má distribuição médica anteriores foi a fixação dos profissionais médicos nas áreas de vulnerabilidade com o profissional intercambista. Os médicos brasileiros demonstraram interesse em ocupar tais vagas, mas há uma alta rotatividade que precisa ser solucionada pelo poder público. Mudanças curriculares nos cursos de graduação, abordando a APS, e o investimento nas estruturas das UBS podem mitigar a rotatividade profissional nas áreas vulneráveis.

Por outro lado, a falta de um monitoramento contínuo da avaliação da qualidade dos formandos e a falta de investimentos na infraestrutura e na regulação da assistência, bem como de melhor distribuição e qualificação dos programas de residência médica, poderão tornar essa situação aparentemente vantajosa uma grande catástrofe (pessoal e profissional), com um mercado de trabalho que pode ter uma enorme oferta de mão de obra pouco qualificada.

\section{Colaboradores}

Nassar LM (0000-0002-4300-4293)* contribuiu para a busca dos artigos e elaboração do texto. Passador JL (0000-0002-0460-8852)* contribuiu para os delineamentos do trabalho e a revisão do texto final. Pereira Júnior GA (0000-0003-3920-3000)* contribuiu para a busca dos artigos, os delineamentos do trabalho e a revisão do texto final. 


\section{Referências}

1. Dal Poz MR. A crise da força de trabalho em saúde. Cad. Saúde Pública. 2013 [acesso em 2021 out 15]; (29):1924-1926. Disponível em: https://www.scielo. br/j/csp/a/F5vfm6dCsVbm9qfngypZ54t/?lang=pt.

2. Simões JC. No Brasil faltam médicos: mito ou realidade? Rev Med Resi. 2011 [acesso em 2021 ou 15]; (13):1-2. Disponível em: http://www.crmpr.org.br/publicacoes/cientificas/index.php/revista-do-medico-residente/article/view/118/118.

3. Santos LMP, Oliveira A, Trindade JR, et al. Implementation research: Towards universal health coverage with more doctors in Brazil. Bull World Health Organ. 2017 [acesso em 2021 out 15]; (95):103-112. Disponível em: https://www.ncbi.nlm.nih.gov/pmc/ articles/PMC5327934/.

4. Scheffer MC, Cassenote A, Guillooux AGA, et al. Demografia Médica no Brasil. São Paulo: FMUSP, CFM, Cremesp; 2018. [acesso em 2021 out 15]. Disponível em: https://jornal.usp.br/wp-content/uploads/DemografiaMedica2018.pdf.

5. Oliveira APC, Gabriel M, Poz MRD, et al. Challenges for ensuring availability and accessibility to health care services under Brazil's Unified Health System (SUS). Ciênc. Saúde Colet. 2017 [acesso em 2021 out 15]; 22:1165-1180. Disponível em: https://pubmed. ncbi.nlm.nih.gov/28444043/.

6. Silveira RS, Pinheiro R. Entendendo a necessidade de médicos no interior da Amazônia - Brasil. Rev bras educ med. 2014 [acesso em 2021 out 15]; (38):451-459. Disponível em: https://www.scielo.br/j/rbem/a/hVW4JjFSJHmxBkpTpCLmsgQ/ abstract/?lang=pt\#: :text=O\%20interior\%20da\%20 Amaz\%C3\%B4nia\%20possui,\%C3\%A9\%20o\%20interior\%20da\%20Amaz\%C3\%B4nia.

7. Brasil. Decreto ${ }^{\circ}$ 53.642, de 28 de Fevereiro de 1964. Dispõe sobre a duplicação de matrículas no primeiro ano das escolas superiores. Diário Oficial da União. 29 Fev 1964.
8. Brasil. Lei ${ }^{0} 12.871$, de 22 de Outubro de 2013. Institui o Programa Mais Médicos, altera as Leis n ${ }^{\circ} 8.745$, de 9 de dezembro de 1993, e $n^{\circ}$ 6.932, de 7 de julho de 1981, e dá outras providências. Diário Oficial da União. 22 Out 2013.

9. Tangcharoensathien V, Mills A, Palu T. Accelerating health equity: The key role of universal health coverage in the Sustainable Development Goals. BMC Med. 2015 [acesso em 2021 out 15]; 13(101). Disponível em: https://bmcmedicine.biomedcentral.com/ articles/10.1186/s12916-015-0342-3.

10. Joanna Briggs Institute. Methodology for JBI Scoping Reviews. [acesso em 2020 jun 6]. Disponível em: https://reviewersmanual.joannabriggs.org/display/MANUAL/Chapter+11\%3A+Scoping+reviews.

11. Povoa L, Andrade MV. Distribuição geográfica dos médicos no Brasil: uma análise a partir de um modelo de escolha locacional. Cad. Saúde Pública. 2006 [acesso em 2021 out 15]; (22):1555-1564. Disponível em: https://www.scielo.br/j/csp/a/DggrwSbWKmrLjJ4zH6TvScy/abstract/?lang=pt.

12. Martins ACP, Sant'anna PA, Rocha JFD, et al. Programa mais médicos: uma revisão integrativa. Rev Bras Promoç Saúde. 2017 [acesso em 2021 out 15]; (30):1-14. Disponível em: https://periodicos.unifor. br/RBPS/article/view/6515.

13. Stralen ACSV, Massote AW, Carvalho CL, et al. Percepção de médicos sobre fatores de atração e fixação em áreas remotas e desassistidas: rotas da escassez. Physis. 2017 [acesso em 2021 out 15]; (22):147-172. Disponível em: https://www.scielo.br/j/physis/a/ f6GHFLTtPjsk8RJHShKGTFw/abstract/?lang=pt.

14. Martinez MR. A abordagem equitativa de gênero como uma estratégia de gestão para fixação de médicos em áreas vulneráveis. Interface. 2017 [acesso em 2021 out 15]; 21:1193-1204. Disponível em: https:// www.scielo.br/j/icse/a/NyzjdrRGV4R8ZNDJJQssXsz/abstract/?lang=pt. 
15. Weber CAT. Dialectics of a medical provision policy in priority areas in Brazil. Rev Assoc Med Bras. 2017 [acesso em 2021 out 15]; (63):268-277. Disponível em: https://www.scielo.br/j/ramb/a/dXxTZ7qjzfMCbr9 sFrfSgBJ/?lang=en.

16. Ezequiel OS, Lucchetti G, Lucchetti ALG, et al. Geographical distribution of medical graduates from a public university. Rev Assoc Med Bras. 2017 [acesso em 2021 out 15]; 63:512-520. Disponível em: https:// www.scielo.br/j/ramb/a/VVtqBLGpYWvYcvs6SP4 tYYF.

17. Silva EM, Ramos MC, Santos W, et al. Cost of providing doctors in remote and vulnerable areas: Programa Mais Médicos in Brazil. Rev Panam Salud Públ. 2018 [acesso em 2021 out 15]; (42):ell. Disponível em: https://iris.paho.org/handle/10665.2/34972.

18. Figueiredo AM, Mckinley DW, Lima KC, et al. Medical school expansion policies: educational access and physician distribution. Med Educ. 2019 [acesso em 2021 out 15]; 53:1121-1131. Disponível em: https:// onlinelibrary.wiley.com/doi/abs/10.1111/medu.13941.

19. Pinto Junior EP, Amorim LDAF, Aquino R. Programa Mais Médicos: contexto de implantação e efeito no provimento de médicos na atenção primária à saúde no Brasil, 2008 a 2016. Rev Panam Salud Publica. 2020 [acesso em 2021 out 15]; (44):e23. Disponível em: https://iris.paho.org/ handle/10665.2/51479\#: :text=O\%20PMM\%20 tem\%20contribu\%C3\%ADdo\%20para,do\%20Sistema\%20\%C3\%9Anico\%20de\%20Sa\%C3\%BAde.

20. Guest RS, Baser R, Li Y, et al. Cancer surgeons' distress and well-being, II: Modifiable factors and the potential for organizational interventions. Ann Surg Oncol. 2011 [acesso em 2021 out 15]; (18):1236-1242. Disponível em: https://pubmed.ncbi.nlm.nih.gov/21399883/.

21. Maciel-Lima SM, Hoffmann-Horochovski MT, Rasia JM. Programa Mais Médicos: Limites e Potencialidades. Rev Direito Bras. 2017 [acesso em 2021 out 15]; (17):291-305. Disponível em: https://pesquisa.bvsalud.org/portal/resource/pt/sus-35929.
22. Instituto Brasileiro de Geografia e Estatística. Censo Demográfico. [acesso em 2020 set 27]. Disponível em: https://www.ibge.gov.br/estatisticas/sociais/ populacao $/ 25089$-censo-1991- 6 .html? =\&t=o-que-e.

23. Conselho Federal de Medicina. Número de hospitais-escola está abaixo das necessidades do sistema formador de futuros profissionais da saúde. 2015 [acesso em 2020 abr 27]. Disponível em: https://portal.cfm. org.br/index.php?option=com $\_$content\&view $=$artic le\&id=25691:2015-08-25-12-47-51\&catid=3.

24. Scheffer MC, Cassenote A, Biancarelli A. Demografia Médica no Brasil. São Paulo: FMUSP, CFM, Cremesp; 2011. [acesso em 2021 out 15]. Disponível em: https://www.cremesp.org.br/pdfs/demografia_medica_brasil_29112011.pdf.

25. Instituto Brasileiro de Geografia e Estatística. Censo Demográfico. Projeção da população do Brasil e das Unidades da Federação. [acesso em 2020 nov 9]. Disponível em: https://www.ibge.gov.br/apps/populacao/projecao//.

26. Ministério da Educação. eMEC. [acesso em 2020 nov 9]. Disponível em: https://emec.mec.gov.br/.

27. Carvalho CHA. O PROUNI no governo Lula e o jogo político em torno do acesso ao ensino superior. Educ Soc. 2006 [acesso em 2021 out 15]; (27):979-1000. Disponível em: https://www.scielo.br/j/es/a/PWLcgtgCgvYP9tXx6NPfsHf/abstract/?lang=pt.

28. Oliveira BLCA, Lima SF, Pereira MUL, et al. Evolução, Distribuição e Expansão dos Cursos de Medicina no Brasil (1808-2018). Trab. Educ. Saúde. 2019 [acesso em 2021 out 15]; (17):e0018317. Disponível em: https://www.scielo.br/j/tes/a/SGBd4Hbk5ghW D3yg6vqt3Jk/?lang=pt\#: :text=Observou\%2Dse\%20 que\%2C\%20entre\%201808,PMM\%20no\%20governo\%20Dilma\%2DTemer.

29. Organização para a Cooperação e Desenvolvimento Econômico. OECD Health Statistics 2013. [acesso em 2020 set 15]. Disponível em: http://dx.doi.org/10.1787/ health-data-en. 
30. Organização para a Cooperação e Desenvolvimento Econômico. Recent Trends in International Migration of Doctors, Nurses and Medical Students, 2019. Paris: OECD Publishing. [acesso em 2020 set 15]. Disponível em: https://dx.doi.org/10.1787/5571ef48-en.

31. Instituto Brasileiro de Geografia e Estatística. Censo Demográfico. IBGE Cidades. [acesso em 2020 nov 9]. Disponível em: https://cidades.ibge.gov.br/brasil/ panorama.

32. Worldometer. Countries in world by population 2020 . [acesso em 2020 nov 9]. Disponível em: https://www. worldometers.info/world-population/population-by-country/.

33. World Directory of Medical Schools. Search the World Directory. [acesso em 2020 nov 9]. Disponível em: https://search.wdoms.org/.

34. Gok SM, Sezen B. Capacity inefficiencies of teaching and non-teaching hospitals. Serv Ind J. 2012 [acesso em 2021 out 15]; 4:2307-2328. Disponível em: https:// www.tandfonline.com/doi/abs/10.1080/02642069.2 011.582495 .

35. Luedy A, Mendes VLS, Júnior HR. Gestão pública por resultados: contrato de gestão como indutor de melhorias em um hospital universitário. Org Soc. 2012 [acesso em 2021 out 15]; 19:641-659. Disponível em: https://periodicos.ufba.br/index.php/revistaoes/article/view/11217.

36. Grosskopfa S, Margaritisb D, Valdmanisc V. The effects of teaching on hospital productivity. Socio-Econ Plan Sci. 2001 [acesso em 2021 out 15]; (35):189-204. Disponível em: https://ideas.repec.org/a/eee/soceps/ v35y2001i3p189-204.html.

37. Brasil. Decreto $n^{\circ}$ 63.341, de $1^{\circ}$ de Outubro de 1968. Estabelece critérios para a expansão do ensino superior e dá outras providências. Diário Oficial da União. 1 Out 1968.

38. Conselho Federal de Medicina. Informe aos Presidentes dos Conselhos Regionais de Medicina. 20. ed. Brasília, DF: CMF; 2020.
39. Conselho Federal de Medicina. Radiografia das Escolas Médicas do Brasil. [acesso em 2020 nov 5]. Disponível em: http://webpainel.cfm.org.br/QvAJAXZfc/ opendoc.htm?document=Radiografia\%20do\%20Ensino\%20m\%C3\%A9dico\%2FRadiografia\%20do\%20 Ensino\%20m\%C3\%A9dico.qvw\&host=QVS\%40scfm 73\&anonymous=true.

40. Telles H, Silva ALA, Bastos C. Programa Mais Médicos do Brasil: a centralidade da relação médico-usuário para a satisfação com o programa. Caderno $\mathrm{CRH}$. 2019 [acesso em 2021 out 15]; (32):101-123. Disponível em: https://periodicos.ufba.br/index.php/crh/ article/view/23470.

41. Santos W, Comes Y, Pereira LL, et al. Avaliação do Programa Mais Médicos: relato de experiência. Saúde debate. 2019 [acesso em 2021 out 15]; 43(120):256268. Disponível em: https://www.scielo.br/j/sdeb/a/ MQMcCdh3qvwpqKQw4vStYqr/abstract/?lang=pt.

42. Rech MRA, Hauser L, Wollmann L, et al. Qualidade da atenção primária no Brasil e associação com o Programa Mais Médicos. Rev Panam Salud Publica. 2018 [acesso em 2021 out 15]; 42:el64. Disponível em: https://scielosp.org/article/rpsp/2018.v42/e164/.

43. Potter H. Falta de profissionais no Mais Médicos afeta 6 milhões de brasileiros. Uol. 2019 jul 22 [acesso em 2020 nov 11]. Disponível em: https://noticias.uol. com.br/ultimas-noticias/deutschewelle/2019/07/22/ falta-de-profissionais-no-mais-medicos-afeta-6-milhoes-de-brasileiros.htm.

44. Folha de São Paulo. Brasileiros chegaram a preencher todas as vagas no Mais Médicos, mas desistência é alta. Folha de São Paulo. 2019 out. [acesso em 2020 nov 11]. Disponível em: https://wwwl.folha.uol. com.br/cotidiano/2019/10/brasileiros-chegaram-a-preencher-todas-as-vagas-no-mais-medicos-mas-desistencia-e-alta.shtml.

45. Giovanella L, Bousquat A, Fausto MCCR, et al. Novos caminhos: tipologia das unidades básicas de saúde brasileiras. Nota Técnica 5/2015. Região e Redes: Caminho da Universalização da Saúde da Saúde no Brasil. [acesso em 2021 out 15]. Disponível em: http:// 
www.resbr.net.br/wp-content/uploads/2015/09/NovosCaminhos05_ValeEste.pdf.

46. Vargas AFM, Vargas DS, Campos MM, et al. The More Doctors Program and the Curricular Guidelines for Medical courses: a comparative analysis among higher education institutions. Interface (Botucatu). 2019 [acesso em 2021 out 15]; (23):e170903. Disponível em: https://www.scielo.br/j/icse/a/bJpqWm BMnTnW5F9bVk9qTQD/?lang=en.
47. Oliveira, FP, Santos LMP, Shimizu HE. Social Accountability of Medical Schools and Social Representations of Medical Students in the Context of the More Doctors Program. Rev Bras Edu Med. 2019 [acesso em 2021 out 15]; 43(1):462-472. Disponível em: https://www.scielo.br/j/rbem/a/rgFdtV6c3cndY564 bSwM93n/?lang=en.

Recebido em 18/01/2021

Aprovado em 09/10/2021

Conflito de interesses: inexistente

Suporte financeiro: não houve 